\title{
SUPPRESSION OF EXCITED-ATOM TWO-PHOTON IONIZATION DUE TO RABI OSCILLATIONS BETWEEN THE EXCITED AND A LOWER STATE
}

\author{
A. Grudka and R. Parzyński \\ Faculty of Physics, Adam Mickiewicz University \\ Umultowska 85, 61-614 Poznań, Poland
}

(Received December 14, 2000)

\begin{abstract}
Optical resonance between the initially occupied excited atomic state and some lower lying state is shown to be a suppressor of two-photon ionization from this excited state via near-threshold Rydberg states because of Rabi oscillations between the resonantly coupled states.

PACS numbers: $32.80 . \mathrm{Rm}, 42.50 . \mathrm{Hz}$
\end{abstract}

\section{Introduction}

It is the aim of this paper to extend our recent model $[1,2]$ of nominal two-photon ionization from an isolated excited state $(n>1)$ through near-threshold Rydberg states $(n \gg 1)$. In the model we studied the effects of migration of population from the directly excited band of high- $n$ Rydberg states towards comparable-energy Rydberg states but of higher angular momenta, due to a sequence of resonant $V$-type Raman couplings $(\Delta l=2)$ exploiting, as resonant intermediaries, the orbitally degenerated states of the same principal quantum number as that of the initial state. It was found in $[1,2]$ that such a migration of population can be responsible for suppression of ionization (the strong-field ionization suppression or stabilization is still a vivid problem [3]). However, one can expect another mechanism of ionization suppression when ionizing the excited state via near-threshold Rydberg states. In this paper we shall show that in such a case the initial-state to a lower-state resonance can be of great importance. To be specific, let the principal quantum number of the initial state be equal to $n=7$. When choosing, for example, the laser frequency in such a way that the initial state is in near-resonance with the states of the principal quantum numbers around $n=40$, then at the same time we produce near-resonance with the lower-lying state of the principal quantum number equal to 5 . What, in fact, we are interested in throughout the 
present paper is to prove that the latter resonance acts as a suppressor of the nominally two-photon ionization from the initial excited state via near-threshold Rydberg states. An estimation of this effect will be made on the basis of the actual hydrogenic parameters.

\section{The model and its solution}

As shown in Fig. 1, we choose the initial state of the hydrogen atom to be the $|n, l, m\rangle=|7,3,2\rangle$ state (black dot). The atom in this state is exposed to the interaction with a laser beam of linear polarization along the $z$-axis. The frequency of the laser beam ensures the atom to be resonantly excited in a one-photon process to the states of the principal quantum numbers around $n=40$. For the frequency chosen, not only the higher states of $n \cong 40$ will be reached in the one-photon process but also the states lying below the initial one, namely the $|5,2,2\rangle$ and $|5,4,2\rangle$ states. Though the lower states are not in general in perfect resonance, the detunings $\left(E_{i}-E_{k}-\hbar \omega\right) / \hbar(i=1, k=3,4)$ can be compensated by the appropriate Rabi frequencies $V_{k i} / \hbar$ in an enough intense field, which makes the transitions to the lower states effective. To treat the problem analytically we make standard approximations, namely, the rotating wave approximation and the dipole

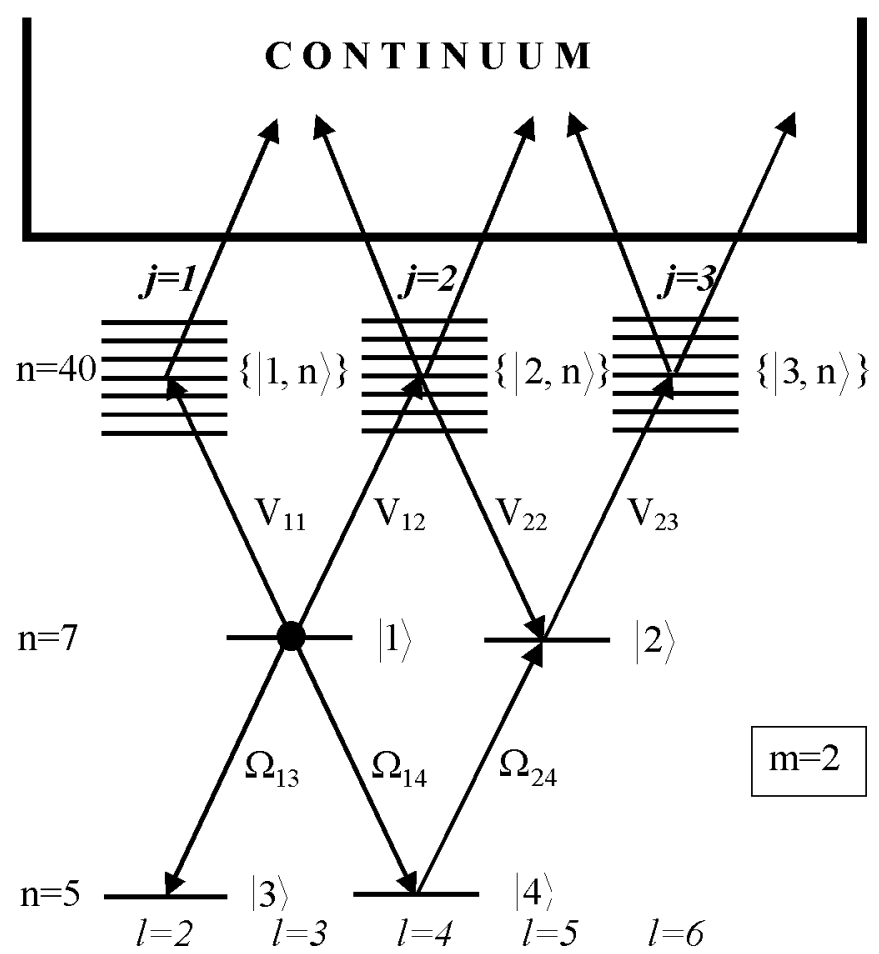

Fig. 1. The ionization from an excited state (black dot) via high-n Rydberg states extended to include resonances between the initial state and some lower states. 
approximation. We also assume a rectangular pulse. Under such circumstances the Schrödinger equation is easily transformed to the Laplace domain (e.g. [4]).

We denote the essential states as follows. Let $|1\rangle=|7,3,2\rangle=|n, l, m\rangle$ stand for the initial state and $|2\rangle$ for the state degenerated with $|1\rangle$ but of the angular quantum number equal to 5. By $|3\rangle$ and $|4\rangle$ we denote the lower states of the principal quantum number equal to 5 and the angular momentum quantum numbers equal to 2 and 4 , respectively. Finally $|j, n\rangle(j=1,2,3)$ stands for high Rydberg states of $n \cong 40$ and the angular quantum number is equal to $2 j$. Because of the selection rules for linear polarization, all states have the same magnetic quantum number equal to that in the initial state, i.e. 2 . By $\Omega_{j j}$ we denote the interaction-Hamiltonian matrix element (from now we use the units in which $\hbar=1$ ) between states $|j\rangle$ and $\left|j^{\prime}\right\rangle$, while $V_{j^{\prime}, j n}$ stands for the matrix element between states $\left|j^{\prime}\right\rangle$ and $|j, n\rangle$. The latter matrix element scales as $n^{-3 / 2}$ [5], i.e. for a large $n$ it is a slowly varying function of $n$. Since in our case the most important up-transitions are to those states whose principal quantum numbers are around $n=40$, we neglect this $n$-dependence introducing some representative matrix element, which we choose to be $V_{j^{\prime}, j n} \cong V_{j^{\prime}, j 40} \equiv V_{j^{\prime} j}$, so equal to that for the states which are in exact resonance. Finally, by $D_{j n, j n^{\prime}}$ we denote the Raman couplings through the continuum between two high-lying Rydberg states of the same angular momentum quantum number. We do not include the Raman couplings via the continuum between Rydberg states of angular momentum numbers differing by 2 because of their negligibility when compared to the resonant couplings through the bound states $|1\rangle$ and $|2\rangle$. As the Raman couplings $D_{j n, j n^{\prime}}$ scale as $\left(n n^{\prime}\right)^{-3 / 2}[6]$, we also neglect their dependence on the principal quantum numbers.

By $b_{j}$ we denote the time-dependent Schrödinger amplitude for the state $|j=1-4\rangle$, and by $b_{j n}$ the amplitude for the state $|j, n\rangle$. The corresponding Laplace transformations $(t \rightarrow s)$ are denoted by $\tilde{b}_{j}$ and $\tilde{b}_{j n}$, respectively. The equations for those transformations, found from the Schrödinger equation in a way analogous to that in [2], have the following form:

$$
\begin{aligned}
& (s-\mathrm{i} \delta) \tilde{b}_{3}=-\mathrm{i} \Omega_{13} \tilde{b}_{1}, \\
& (s-\mathrm{i} \delta) \tilde{b}_{4}=-\mathrm{i} \Omega_{14} \tilde{b}_{1}-\mathrm{i} \Omega_{24} \tilde{b}_{2}, \\
& s \tilde{b}_{1}=-\mathrm{i} \Omega_{13} \tilde{b}_{3}-\mathrm{i} \Omega_{14} \tilde{b}_{4}-\mathrm{i} V_{11} K_{1}-\mathrm{i} V_{12} K_{2}+1, \\
& s \tilde{b}_{2}=-\mathrm{i} \Omega_{24} \tilde{b}_{4}-\mathrm{i} V_{22} K_{2}-\mathrm{i} V_{23} K_{3}, \\
& \left(s-\mathrm{i} \Delta_{n}\right) \tilde{b}_{1 n}=-\mathrm{i} V_{11} \tilde{b}_{1}-D_{1} K_{1}, \\
& \left(s-\mathrm{i} \Delta_{n}\right) \tilde{b}_{2 n}=-\mathrm{i} V_{12} \tilde{b}_{1}-\mathrm{i} V_{22} \tilde{b}_{2}-D_{2} K_{2}, \\
& \left(s-\mathrm{i} \Delta_{n}\right) \tilde{b}_{3 n}=-\mathrm{i} V_{23} \tilde{b}_{2}-D_{3} K_{3},
\end{aligned}
$$


where $K_{j}=\sum_{n} \tilde{b}_{j n}, \delta$ is a detuning of the laser frequency from the frequencies of the atomic transitions $|1\rangle \rightarrow|3\rangle,|4\rangle$ and $|2\rangle \rightarrow|4\rangle, \Delta_{n}$ is a detuning for the $|1\rangle \rightarrow|j, n\rangle$ and $|2\rangle \rightarrow|j, n\rangle$ transitions, and $s$ is the Laplace variable.

To find the solution, we first divide equations (5-7) by $s-\mathrm{i} \Delta_{n}$ and then sum the result over $n$, obtaining

$$
\begin{aligned}
& K_{1}=-\mathrm{i} V_{11} P \tilde{b}_{1}-P D_{1} K_{1}, \\
& K_{2}=-\mathrm{i} V_{12} P \tilde{b}_{1}-\mathrm{i} V_{22} P \tilde{b}_{2}-P D_{2} K_{2}, \\
& K_{3}=-\mathrm{i} V_{23} P \tilde{b}_{2}-P D_{3} K_{3},
\end{aligned}
$$

where $P=\sum_{n}\left(s-\mathrm{i} \Delta_{n}\right)^{-1}$. Now, using Eqs. (3) and (4) and (8-10), we express $\tilde{b}_{3}$ and $\tilde{b}_{4}$ by $\tilde{b}_{1}$ and $\tilde{b}_{2}$. Next, substituting these expressions into formulae (1) and (2) we get a set of algebraic equations for the amplitudes $\tilde{b}_{1}$ and $\tilde{b}_{2}$

$$
\begin{aligned}
& a(s) \tilde{b}_{1}+b(s) \tilde{b}_{2}=s-\mathrm{i} \delta \\
& b(s) \tilde{b}_{1}+c(s) \tilde{b}_{2}=0
\end{aligned}
$$

where

$$
\begin{aligned}
& a(s)=\left[(s-\mathrm{i} \delta) s+(s-\mathrm{i} \delta)\left(\frac{V_{11}^{2} P}{1+P D_{1}}+\frac{V_{12}^{2} P}{1+P D_{2}}\right)+\Omega_{13}^{2}+\Omega_{14}^{2}\right], \\
& b(s)=\left[(s-\mathrm{i} \delta) \frac{V_{12} V_{22} P}{1+P D_{2}}+\Omega_{14} \Omega_{24}\right], \\
& c(s)=\left[(s-\mathrm{i} \delta) s+(s--\mathrm{i} \delta)\left(\frac{V_{22}^{2} P}{1+P D_{2}}+\frac{V_{23}^{2} P}{1+P D_{3}}\right)+\Omega_{24}^{2}\right] .
\end{aligned}
$$

From this set we obtain

$$
\begin{aligned}
& \tilde{b}_{1}=\frac{(s-\mathrm{i} \delta) c(s)}{W(s)} \\
& \tilde{b}_{2}=-\frac{(s-\mathrm{i} \delta) b(s)}{W(s)}
\end{aligned}
$$

where

$$
W(s)=a(s) c(s)-b(s)^{2} .
$$

For mathematical simplicity, we adopt the Bixon-Joertner structure [7], i.e. assume, due to a high $n$, an equal spacing of states around the state of $n=40$. In such a case $\Delta_{n}=(n-40) \Delta$, where $\Delta$ is the energy difference between the neighboring states of $n$ equal to 40 and 41 . In such a case, the summation inherent in $P$ converges to $(\pi / \Delta)(1+\mu) /(1-\mu)$, with $\mu=\exp (-2 \pi s / \Delta)$ [8]. The standard way to find the inverse Laplace transformation is to expand $P$ in a power series of $\mu$. It is known [2] that, retaining the zero-order term only, we restrict the time scale to pulses shorter than $2 \pi / \Delta$, i.e. the characteristic Kepler period for $n=40$. Assuming that only such pulses are of our interest we replace $P$ by $\pi / \Delta$ in all equations. By the elementary fraction decomposition, we then convert Eqs. (16) and (17) into

$$
\tilde{b}_{i}=\sum_{j=1}^{4} \operatorname{Res} \tilde{b}_{i}\left(s_{j}\right) \frac{1}{s-s_{j}} \quad(i=1,2)
$$


where the sum runs over all poles of $\tilde{b}_{i}$ with respect to $s$. As a result, the inverse Laplace transformation is

$$
b_{i}=\sum_{j=1}^{4} \operatorname{Res} \tilde{b}_{i}\left(s_{j}\right) \exp \left(s_{j} t\right) \quad(i=1,2) .
$$

To find $b_{3}, b_{4}$, and $b_{j n}(j=1,2,3)$ we substitute (19) into (1), (2), and (5-7), using in the latter case Eqs. (8-10). Along this line one gets

$$
\begin{aligned}
& \tilde{b}_{3}=-\mathrm{i} \sum_{j=1}^{4} \Omega_{13} \operatorname{Res} \tilde{b}_{1}\left(s_{j}\right) \frac{1}{s-s_{j}} \frac{1}{s-\mathrm{i} \delta}, \\
& \tilde{b}_{4}=-\mathrm{i} \sum_{j=1}^{4}\left[\Omega_{14} \operatorname{Res} \tilde{b}_{1}\left(s_{j}\right)+\Omega_{24} \operatorname{Res} \tilde{b}_{2}\left(s_{j}\right)\right] \frac{1}{s-s_{j}} \frac{1}{s-\mathrm{i} \delta}, \\
& \tilde{b}_{1 n}=\frac{-\mathrm{i}}{1+P D_{1}} \sum_{j=1}^{4} V_{11} \operatorname{Res} \tilde{b}_{1}\left(s_{j}\right) \frac{1}{s-s_{j}} \frac{1}{s-\mathrm{i} \Delta_{n}}, \\
& \tilde{b}_{2 n}=\frac{-\mathrm{i}}{1+P D_{2}} \sum_{j=1}^{4}\left[V_{12} \operatorname{Res} \tilde{b}_{1}\left(s_{j}\right)+V_{22} \operatorname{Res} \tilde{b}_{2}\left(s_{j}\right)\right] \frac{1}{s-s_{j}} \frac{1}{s-\mathrm{i} \Delta_{n}}, \\
& \tilde{b}_{3 n}=\frac{-\mathrm{i}}{1+P D_{3}} \sum_{j=1}^{4} V_{23} \operatorname{Res} \tilde{b}_{2}\left(s_{j}\right) \frac{1}{s-s_{j}} \frac{1}{s-\mathrm{i} \Delta_{n}} .
\end{aligned}
$$

These equations are then transformed to the time domain by applying the Borel convolution theorem. For a given product $\tilde{f} \tilde{g}$ of two Laplace transformations, with $\tilde{f} \rightarrow f$ as well as $\tilde{g} \rightarrow g$, the inverse Laplace transformation of $\tilde{f} \tilde{g}$ is equal to the convolution $f * g$, namely

$$
\tilde{f} \tilde{g} \rightarrow f * g=\int_{0}^{t} f\left(t^{\prime}\right) g\left(t-t^{\prime}\right) \mathrm{d} t^{\prime} .
$$

Choosing $\tilde{f}$ as $\left(s-s_{j}\right)^{-1}$ and $\tilde{g}$ as $(s-\mathrm{i} \delta)^{-1}$ or $\left(s-\mathrm{i} \Delta_{n}\right)^{-1}$, one finds $f * g$ equal to $\exp (\mathrm{i} \delta t) f\left(s_{j}-\mathrm{i} \delta, t\right)$ or $\exp \left(\mathrm{i} \Delta_{n} t\right) f\left(s_{j}-\mathrm{i} \Delta_{n}, t\right)$, respectively, where $f(x, t)=[\exp (x t)-1] / x$. The use of the convolution theorem results in the following time-dependent amplitudes:

$$
\begin{aligned}
& b_{3}=-\mathrm{i} \exp (\mathrm{i} \delta t) \sum_{j=1}^{4} \Omega_{13} \operatorname{Res} \tilde{b}_{1}\left(s_{j}\right) f\left(s_{j}-\mathrm{i} \delta, t\right), \\
& b_{4}=-\mathrm{i} \exp (\mathrm{i} \delta t) \sum_{j=1}^{4}\left[\Omega_{14} \operatorname{Res} \tilde{b}_{1}\left(s_{j}\right)+\Omega_{24} \operatorname{Res} \tilde{b}_{2}\left(s_{j}\right)\right] f\left(s_{j}-\mathrm{i} \delta, t\right), \\
& b_{1 n}=\frac{-\mathrm{i} \exp \left(\mathrm{i} \Delta_{n} t\right)}{1+P D_{1}} \sum_{j=1}^{4} V_{11} \operatorname{Res} \tilde{b}_{1}\left(s_{j}\right) f\left(s_{j}-\mathrm{i} \Delta_{n}, t\right),
\end{aligned}
$$




$$
\begin{aligned}
& b_{2 n}=\frac{-\mathrm{i} \exp \left(\mathrm{i} \Delta_{n} t\right)}{1+P D_{2}} \sum_{j=1}^{4}\left[V_{12} \operatorname{Res} \tilde{b}_{1}\left(s_{j}\right)+V_{22} \operatorname{Res} \tilde{b}_{2}\left(s_{j}\right)\right] f\left(s_{j}-\mathrm{i} \Delta_{n}, t\right), \\
& b_{3 n}=\frac{-\mathrm{i} \exp \left(\mathrm{i} \Delta_{n} t\right)}{1+P D_{3}} \sum_{j=1}^{4} V_{23} \operatorname{Res} \tilde{b}_{2}\left(s_{j}\right) f\left(s_{j}-\mathrm{i} \Delta_{n}, t\right) .
\end{aligned}
$$

Taking square modulus of $b_{j n}(j=1,2,3)$, and then summing the result over $n$, one gets the whole population $W_{j}$ in a given band of high- $n$ Rydberg states of the angular momentum quantum number $l=2 j,(j=1,2,3)$

$$
\begin{aligned}
W_{1}= & \frac{2 \pi}{\Delta}\left|\frac{1}{1+P D_{1}}\right|^{2} \sum_{j, k=1}^{4}\left[V_{11} \operatorname{Res} \tilde{b}_{1}\left(s_{j}\right)\right]\left[V_{11} \operatorname{Res} \tilde{b}_{1}\left(s_{k}\right)\right]^{*} f\left(s_{j}+s_{k}^{*}, t\right), \\
W_{2}= & \frac{2 \pi}{\Delta}\left|\frac{1}{1+P D_{2}}\right|^{2} \sum_{j, k=1}^{4}\left[V_{12} \operatorname{Res} \tilde{b}_{1}\left(s_{j}\right)+V_{22} \operatorname{Res} \tilde{b}_{2}\left(s_{j}\right)\right] \\
& \times\left[V_{12} \operatorname{Res} \tilde{b}_{1}\left(s_{k}\right)+V_{22} \operatorname{Res} \tilde{b}_{2}\left(s_{k}\right)\right]^{*} f\left(s_{j}+s_{k}^{*}, t\right), \\
W_{3}= & \frac{2 \pi}{\Delta}\left|\frac{1}{1+P D_{3}}\right|^{2} \sum_{j, k=1}^{4}\left[V_{23} \operatorname{Res} \tilde{b}_{2}\left(s_{j}\right)\right]\left[V_{23} \operatorname{Res} \tilde{b}_{2}\left(s_{k}\right)\right]^{*} f\left(s_{j}+s_{k}^{*}, t\right) .
\end{aligned}
$$

To find the ionization probability we need to substract from unity the population of all bound states $\left(1-\sum_{j=1}^{4}\left|b_{j}\right|^{2}-\sum_{j=1}^{3} W_{j}\right)$.

\section{Results}

The solutions obtained (Eqs. (20), (27-34)) are valid for times not exceeding $2 \pi / \Delta$, where $\Delta$ is the frequency difference between the states with the principal quantum numbers $n=40$ and $n=41$, i.e. for $t<10^{-11} \mathrm{~s}$. The bound-bound and bound-free matrix elements used were calculated on the basis of the Laplace-transformation technique of Feldman et al. [9]. As we checked for couplings between a low-lying state $(n=7)$ and high-lying ones of $n \gg 1$, the matrix elements exhibited weak $n$ dependence in agreement with the quasiclassical approximation [5, 6]. So the neglect of this dependence, as it was done by us at the beginning, was justified. Furthermore, the Raman coupling between any two Rydberg states of the same angular momentum quantum number was taken in the form $D_{j n, j n^{\prime}}=$ $\left(1+\mathrm{i} q_{j n, j n^{\prime}}\right) \sqrt{\gamma_{j n} \gamma_{j n^{\prime}}} / 2$, where $\gamma_{j n}\left(\gamma_{j n^{\prime}}\right)$ is the Fermi golden rule ionization rate for the state $|j, n\rangle\left(\left|j, n^{\prime}\right\rangle\right)$, and $q_{j n, j n^{\prime}}$ is the Fano parameter [10]. In analogy to [2], the representative Fano parameter was taken equal to 20.

In Figs. 2 and 3 we show representative time evolutions of the bound-state populations and ionization calculated for laser beam intensity of $5 \times 10^{8} \mathrm{~W} / \mathrm{cm}^{2}$. Figure 2 presents time evolutions for the isolated bound states $1,2,3$, and 4 . We see 
a well-pronounced Rabi oscillation of the population between states $|1\rangle$ and $|3\rangle$. As a matter of fact, these states are not in perfect resonance, but the corresponding Rabi frequency $\Omega_{13}$ is bigger than the detuning from the $|1\rangle-|3\rangle$ resonance for the intensity of interest, so the Rabi frequency compensates the detuning. In the graphs for $\left|b_{1}\right|^{2}$ we also show the curve obtained when neglecting the $n=7 \rightarrow 5$ resonance. The striking difference between the corresponding curves, one obtained with and the other obtained without the $7 \rightarrow 5$ resonance, is a direct indicator of the importance of the $n=7 \rightarrow 5$ resonance. Figure 2 also shows a flow of the population through the states $|4\rangle$ and $|2\rangle$ (the lack of the Rabi oscillation between these states is due to much smaller Rabi frequencies when compared to the $\Omega_{13}$ one).
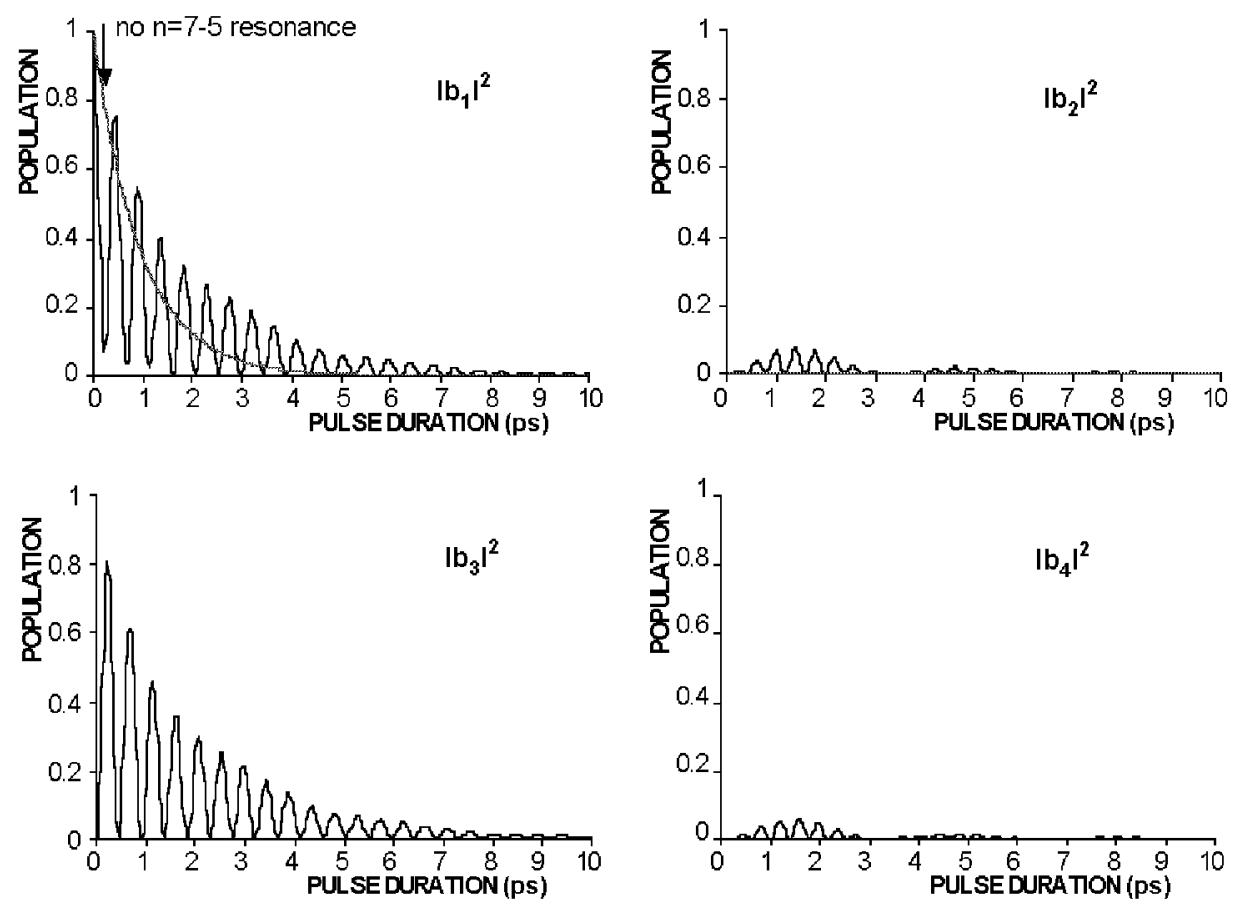

Fig. 2. Time evolutions of the population in the bound states $|1\rangle,|2\rangle,|3\rangle$, and $|4\rangle$ at the laser beam intensity of $5 \times 10^{8} \mathrm{~W} / \mathrm{cm}^{2}$.

Figure 3 shows the evolutions of the populations in high- $n$ Rydberg bands and in the atomic continuum. The most significant population is found to be in the Rydberg states denoted by $|2, n\rangle$ since the matrix elements between the states $|1\rangle$ and $|2, n\rangle$ are higher than those between the states $|1\rangle$ and $|1, n\rangle$. The curves for $W_{j}$ exhibit the negative effect of the $n=7 \rightarrow 5$ resonance on the total population of a given-l Rydberg bands. As to the ionization, the most important difference between the two cases, one including and the other neglecting the $n=7 \rightarrow 5$ resonance, is a substantial decrease in the ionization probability for times shorter than 5 ps when transitions to lower states are possible. The reason for this is that, 

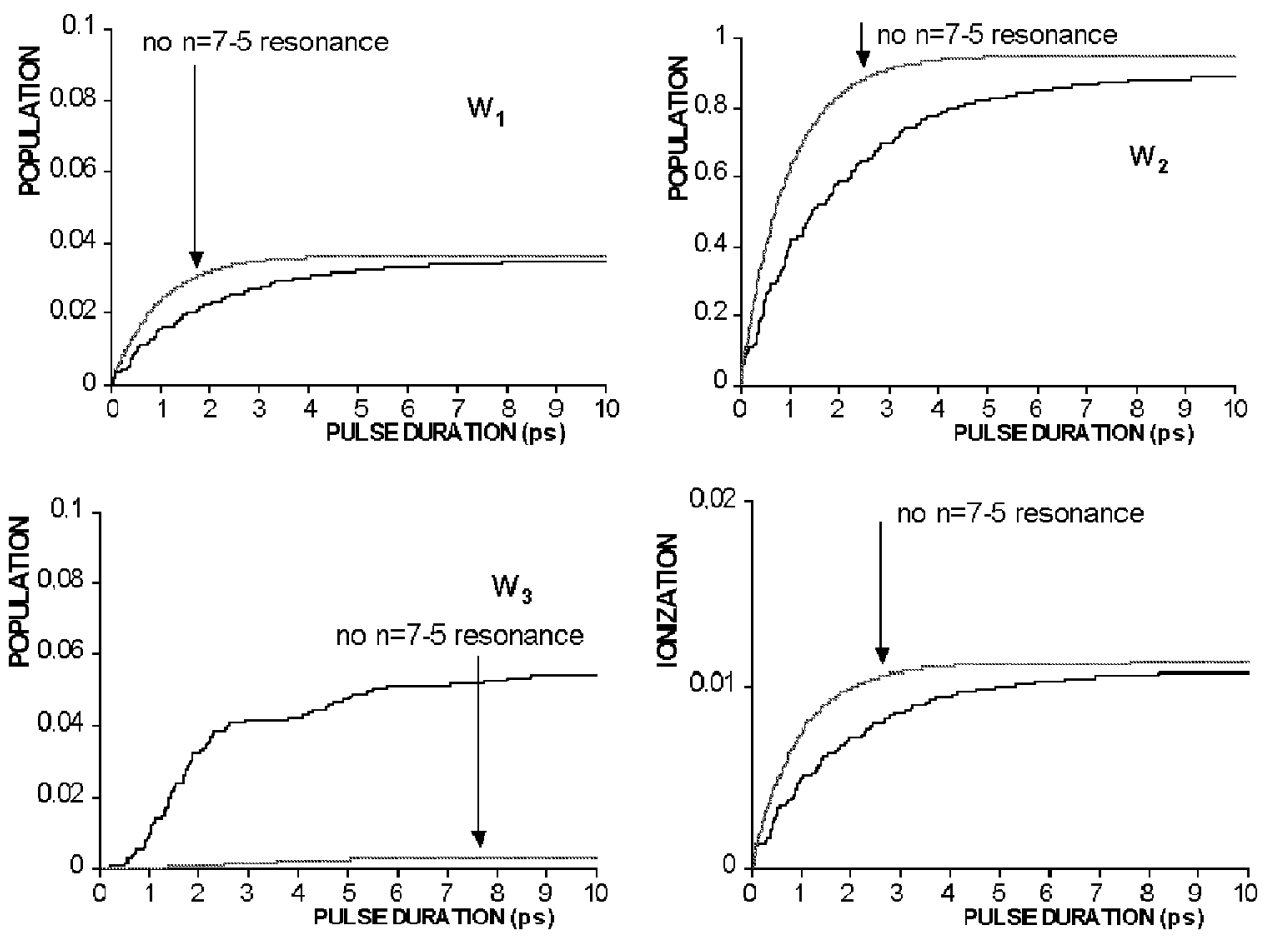

Fig. 3. Time evolutions of the population in the high- $n(n \cong 40)$ Rydberg bands $\left(W_{j}\right)$ and in the continuum at the laser beam intensity of $5 \times 10^{8} \mathrm{~W} / \mathrm{cm}^{2}$.

due to the Rabi oscillation between the initial $|1\rangle$ and the low-lying $|3\rangle$ states, a part of the population is removed from the $|1\rangle$ state which is to be two-photon ionized via high Rydberg states.

\section{Conclusions}

The extension of our previous model $[1,2]$ of nominal two-photon ionization from an excited atomic state via near-threshold Rydberg states has led us to the conclusion that a possible resonance between the initial excited state and some lower-lying state can act as a suppressor of the ionization mentioned. This mechanism of the ionization suppression is an additional mechanism as compared to the one previously discussed by us $[1,2]$ consisting in resonant Raman migration of the population from the directly excited near-threshold Rydberg states towards comparable-energy Rydberg states of higher angular momenta. The present paper shows in a way different than in [11] that, in the ionization starting from an excited state, the lower-lying states can profoundly affect the scenario of the process at appropriate frequencies. 


\section{Acknowledgments}

One of us (A.G.) and the other one (R.P.) wish to thank the Committee for Scientific Research for its support under grants No. 2 P03B 01718 and No. 2 P03B 02619.

\section{References}

[1] A. Wójcik, R. Parzyński, A. Grudka, Phys. Rev. A 55, 2144 (1997).

[2] R. Parzyński, A. Grudka, Phys. Rev. A 58, 1335 (1998).

[3] Super-Intense Laser-Atom Pysics IV, Eds. H.G. Muller, M.V. Fedorov, NATO ASI Series 3 on High Technology, Vol. 13, Kluwer Academic, Dordrecht (The Netherlands) 1996.

[4] S.M. Barnett, P.M. Radmore, Methods in Theoretical Quantum Optics, Clarendon Press, Oxford 1997, p. 27.

[5] N.B. Delone, S.P. Goreslavsky, V.P. Krainov, J. Phys. B 27, 4403 (1994).

[6] M.S. Adams, M.V. Fedorov, V.P. Krainov, D.D. Meyerhofer, Phys. Rev. A 52, 125 (1994).

[7] H.G. Muller, L.D. Noordam, Phys. Rev. Lett. 82, 5024 (1999).

[8] G.C Stey, R.W. Gibberd, Physica 60, 1 (1972).

[9] G. Feldman, T. Fulton, B.R. Judd, Phys. Rev. A 51, 2762 (1995).

[10] R. Grobe, G. Leuchs, K. Rzążewski, Phys. Rev. A 34, 1188 (1986).

[11] J.H. Hoogenraad, R.B. Vrijen, P.W. van Amersfoort, A.F.G. Van der Meer, L.D. Noordam, Phys. Rev. Lett. 75, 4579 (1995). 